\title{
Absolute Eosinophil Count May be an Optimal Peripheral Blood Marker to Identify Risk of Immune- Related Adverse Events in Advanced Malignant Tumors Treated with PD-1/PD-L1 Inhibitors: A Retrospective Analysis.
}

\section{Yan Ma}

Capital Medical University Affiliated Beijing Friendship Hospital

Xiao Ma

Capital Medical University Affiliated Beijing Friendship Hospital

Jingting Wang

Capital Medical University Affiliated Beijing Friendship Hospital

Shanshan Wu

Capital Medical University Affiliated Beijing Friendship Hospital

Jing Wang

Capital Medical University Affiliated Beijing Friendship Hospital

Bangwei Cao ( $\sim$ oncology@ccmu.edu.cn )

Capital Medical University Affiliated Beijing Friendship Hospital https://orcid.org/0000-0001-61642291

\section{Research}

Keywords: Immunotherapy, immune-related adverse events, neutrophil-lymphocyte ratio, plateletlymphocyte ratio, absolute eosinophil count, predictive markers

Posted Date: October 25th, 2021

DOl: https://doi.org/10.21203/rs.3.rs-917475/v2

License: (c) (1) This work is licensed under a Creative Commons Attribution 4.0 International License. Read Full License 


\section{Abstract \\ Background}

This study aimed to investigate the predictive values of serum biomarkers including absolute eosinophil count (AEC), neutrophil-lymphocyte ratio (NLR), and platelet-lymphocyte ratio (PLR) with respect to immune-related adverse events (irAEs) during anti-PD-1/PD-L1 inhibitor treatment in patients with advanced malignant tumors.

\section{Methods}

We retrospectively analyzed 95 patients with advanced cancer who were treated with anti-PD-1/PD-L1 inhibitors from January 1, 2017 to May 1, 2020, in our cancer center. We then analyzed associations between irAEs and anti-PD-1/PD-L1 inhibitor responses and evaluated the predictive values of serum biomarkers with respect to risk of irAEs.

\section{Results}

The incidence of irAEs was 55.8\%. There were no statistically significant differences between the irAEs and no-irAEs groups in objective response rate (ORR) or disease control rate (DCR). However, landmark analysis showed that the irAEs group had better survival after 120 days following the initiation of anti-PD1/PD-L1 inhibitor treatment, compared with the no-irAEs group. The incidences of irAEs were greater in the high-AEC and low-NLR groups than in the low-AEC and high-NLR groups. Univariate logistic analysis showed that low NLR, ECOG performance status $(0-1)$, and high AEC were risk factors for irAEs. Multivariate logistic analysis showed that high AEC and good ECOG performance status were independent predictors for irAEs.

\section{Conclusions}

irAEs may be associated with a survival benefit. Baseline AEC is a strong predictor of irAEs in patients undergoing treatment with anti-PD-1/PD-L1 inhibitors.

\section{Introduction}

Immune checkpoint inhibitor therapy, represented by PD-1/PD-L1, has been widely used for the treatment of many advanced malignant tumors, with significant and sustained efficacy; it has had a robust impact on traditional treatment modalities such as cytotoxic chemotherapy and targeted therapy [1-5]. Despite its favorable effect, the immune-related adverse reactions that occur during immune checkpoint inhibitor treatment should not be ignored. 
irAEs are broadly defined as instances of immune-mediated host organ dysfunction caused by abnormal immune system activity following immunotherapy [6]. They are most common in the skin, thyroid, and gastrointestinal tract, although they may involve any organ or system, including the heart, lungs, liver, and pituitary gland [7]. Immune-related adverse events (irAEs) are usually easy to manage; however, approximately $10 \%$ of affected patients experience irAEs sufficiently severe to require discontinuation of immune checkpoint inhibitor therapy and/or additional treatment with hormonal or immunosuppressive agents $[8,9]$. In some instances, irAEs can lead to permanent illness, with potential fatality in approximately $1 \%$ of affected patients [10]. It is important to note that irAEs can occur at any point in time, including months after treatment withdrawal [11].

Considering these characteristics of irAEs, their diagnosis and prediction are particularly challenging. Peripheral blood markers such as absolute eosinophil count (AEC), neutrophil-lymphocyte ratio (NLR), and platelet-lymphocyte ratio (PLR) have attracted substantial attention because of their non-invasive, rapid, stable, and inexpensive characteristics. NLR and PLR can reportedly predict irAE occurrence related to anti-PD-1/PD-L1 inhibitor therapy in patients with non-small cell lung cancer $[12,13]$. An increased NLR has been associated with an increased risk of grades 3-4 pulmonary and gastrointestinal irAEs in melanoma patients treated with nivolumab [14]. Moreover, eosinophils in peripheral blood were also associated with irAEs [15], and increased eosinophil counts at baseline and 1 month were associated with an increased overall risk of irAEs grade 2 and above [14]. Baseline characteristics of high AEC (0.125 $\times 10^{9} / \mathrm{L}$ ) were associated with an increased risk of immune-associated pneumonia, as well as better clinical outcomes in patients with non-small cell lung cancer (NSCLC) who were receiving immune checkpoint inhibitor treatment [16].

Thus far, the relationships between irAEs and anti-PD-1/PD-L1 inhibitor treatment responses remain controversial. irAEs were positively correlated with anti-PD-1/PD-L1 inhibitor efficacy in patients with NSCLC and patients with melanoma [17-21], but there has been some speculation that this correlation is not robust [22, 23]; a negative correlation has been suggested in patients with small cell lung cancer [24]. Recently, a study by Rogado et al. involving multiple tumor species showed that irAEs were directly associated with favorable objective response rates (ORRs) and progression-free survival in patients receiving anti-PD-1/PD-L1 inhibitors [25].

This study was performed to evaluate the relationships of irAEs with the clinical efficacy of anti-PD-1/PDL1 inhibitors in the treatment of advanced malignant tumors. It also screened predictors of irAE risk by investigation of peripheral blood biomarkers (e.g., baseline AEC and baseline NLR).

\section{Patients And Methods}

\subsection{Study design and patient population}

This retrospective study included patients with malignant tumors who were admitted to the Cancer Center of Beijing Friendship Hospital affiliated with Capital Medical University from January 1, 2017 to May 1, 
2020. All patients had generally complete case data that allowed assessment of efficacy, disease progression or treatment failure, and irAEs. All tumors were pathologically confirmed. The follow-up period began at the initiation of anti-PD-1/PD-L1 inhibitor therapy and ended at the time of disease progression, confirmed death, or August 31,2020. The following exclusion criteria were used: previous medical history or test results indicating the presence of a definite hereditary disease; autoimmune disease or other serious medical conditions, such as cardiovascular disease (e.g., atrioventricular block, atrial fibrillation, or congestive heart failure) or kidney disease (e.g., hemodialysis); failure to evaluate serological indicators; and absence of serological test results recorded before and after treatment. Thus, this study included 95 patients. Anti-PD-1/PD-L1 inhibitors used for treatment in this study mainly included nivolumab, atezolizumab, sintilimab, and camrelizumab. Immunotherapycombined treatment was administered concurrently with either targeted therapy or chemotherapy.

The study protocol was approved by Beijing Friendship Hospital's Institutional Review Board (2020-P2176-01) and performed in accordance with the tenets of the Declaration of Helsinki.

\subsection{Data collection}

Characteristics and clinical data of all 95 patients treated with anti-PD-1/PD-L1 inhibitors were recorded, including age, sex, Eastern Cooperative Oncology Group performance status (ECOG PS), tumor type, cancer TNM staging, treatment lines, treatment, clinical efficacy, and PFS. The tumor stage was determined in accordance with the eighth edition of the Union for International Cancer Control (UICC) TNM classification of Malignant Tumors.

CT scans were performed at baseline, as well as after one and two cycles of anti-PD-1/PD-L1 inhibitor treatment or when clinical disease progression was evident. Response to anti-PD-1/PD-L1 therapy was determined using the Response Evaluation Criteria In Solid Tumors (RECIST) criteria, version 1.1. Efficacy was assessed as complete response (CR), partial response (PR), stable disease (SD), and progressive disease (PD). CR and PR refer to ORR;CR, PR, and SD refer to disease control rate (DCR). PFS was recorded from the beginning of treatment until the observation of disease progression or death from any cause.

irAEs were defined as adverse events with a potential immunologic basis that required close monitoring and/or potential intervention with hormonal or immunosuppressive therapies [20]. irAEs were recorded by analysis of medical records, as well as follow-up interviews involving patients and attending physicians. Baseline measurements were defined as the measurements taken within 3 days prior to receipt of antiPD-1/PD-L1 inhibitor treatment. Baseline peripheral blood data included absolute neutrophil count, absolute lymphocyte count, platelet count, and absolute eosinophil count.

\subsection{Statistical analysis}

All data were statistically analyzed using SPSS Statistics, version 25.0. Forest plots were drawn with $\mathrm{R}$ software, version 4.0.2. Receiver operating characteristic (ROC) curve analysis was used to determine the 
optimal cutoff values for peripheral blood markers. The chi-squared test was in $2 \times 2$ tables. Survival curves were estimated by the Kaplan-Meier method, then analyzed using the log-rank test. Landmark analysis was adopted because of the immortal time bias for irAEs. Associations of baseline AEC, NLR, and PLR with irAEs were evaluated by univariate and multivariate logistic regression analysis. $P<0.05$ was considered statistically significant for all analyses.

\section{Results}

\subsection{Patient characteristics}

All 95 patients received anti-PD-1/PD-L1 inhibitor treatment. The patient characteristics are summarized in Table 1. The median age was 62 years (range, 30-80 years); most patients (64.2\%) exhibited an ECOG PS of 1. First-line and second-line treatments with anti-PD-1/PD-L1 therapy were recorded in $65 \%$ of patients. The median PFS was 108 days. There were zero cases of CR ( $0 \%), 12$ cases of PR $(12.6 \%), 49$ cases of SD (51.6\%), and 34 cases of PD (35.8\%). The ORR and DCR were $12.6 \%$ and $64.2 \%$, respectively.

The incidence of irAEs was $55.8 \%$. Rash, immune-associated pneumonia, and hepatotoxicity were present in eight, seven, and 11 patients, respectively (Table 2).

\subsection{Associations between irAEs and anti-PD-1/PD-L1 inhibitor responses}

ORR in the irAEs and no-irAEs groups were $13.2 \%$ and $11.9 \%$, respectively; corresponding DCRs were $60.4 \%$ and $69.0 \%$. There were no significant differences in ORR and DCR between the two groups $(P=$ 0.763 and $\mathrm{P}=0.381$; Table 3 ).

Considering the immortal time bias of irAEs, PFS was studied using landmark analysis (Fig. 1). Using 120 days as a threshold, the survival data were divided into two sections for survival analysis and a KaplanMeier curve was generated. The risk of disease progression was 0.981 -fold in the irAEs group, compared with the risk in the no-irAEs group; there was no significant difference in PFS between the two groups $(P=$ 0.951). After 120 days, the risk of disease progression was 0.398 -fold in the irAEs group, compared with the risk in the no-irAEs group; the PFS was better in the irAEs group than in the no-irAEs group $(P=0.030)$.

\subsection{Peripheral blood predictive markers for irAEs}

Using irAEs as the result variable, we generated ROC curves of NLR, PLR, and AEC; the respective cutoff values selected were $8.58,180.68$, and $0.045 \times 10^{9} / \mathrm{L}$. Based on cutoff value grouping, we compared the incidences of irAEs among groups; the incidences of irAEs were higher in the low NLR group (59.3\%) than in the high NLR group (22.2\%; $P=0.041$; Table 3$)$. In addition, the incidence of irAEs was significantly higher in the high-AEC group (63.0\%) than in the low-AEC group (31.8\%; P = 0.010; Table 4).

\subsection{Univariate and multivariate logistic analysis of predictive markers for irAEs}


The results of univariate and multivariate logistic analyses are shown in Table 5. In univariate logistic analysis, good ECOG score (0-1), low-NLR (cutoff value: 8.58), and high-AEC (cutoff value: 0.045 $\left.\times 10^{9} / \mathrm{L}\right)$ were important predictors of irAEs $(P=0.0499$, OR: $0.196,95 \% \mathrm{Cl}: 0.038-1.000 ; P=0.0499$, OR: $0.507,95 \% \mathrm{Cl}: 0.241-1.065 ; \mathrm{P}=0.012, \mathrm{OR}: 3.651,95 \% \mathrm{Cl}: 1.322-10.076)$. Multivariate logistic analysis was performed involving factors with $\mathrm{P}<0.2$ in univariate analysis and tumor species; high-AEC and good ECOG score were independently associated with irAEs ( $P=0.014$, OR: 4.114, 95\% Cl: 1.328-12.858; $\mathrm{P}=0.046$, OR: $0.159,95 \% \mathrm{Cl}: 0.026-0.970)$. In addition, immunotherapy combined with targeted therapy was associated with a greater risk of irAEs, compared with other treatments $(P=0.005,0 R: 0.156,95 \% \mathrm{Cl}$ : $0.045-0.544)$.

\subsection{Forest plot of multivariate logistic regression analyses for irAEs}

To more intuitively depict the results of multivariate logistic analysis of irAEs, we generated a forest plot with irAEs as the study event (Fig. 2). .The incidence of irAEs was higher in the high-AEC group than in the low-AEC group; the incidence of irAEs was greater in the good ECOG PS (0-1) group than in the ECOG PS (2) group. Similarly, the incidence of irAEs was lower in patients receiving immunotherapy combined with targeted therapy, compared with patients receiving other treatments.

\subsection{Associations of baseline AEC with anti-PD-1/PD-L1 efficacy}

The ORRs of the high-AEC $\left(>0.045 \times 10^{9} / \mathrm{L}\right)$ and low-AEC $\left(\leq 0.045 \times 10^{9} / \mathrm{L}\right)$ groups were $9.5 \%$ and $22.7 \%$, respectively; the corresponding DCRs were $64.3 \%$ and $63.6 \%$. There were no significant differences in ORR $(P=0.208)$ or DCR $(P=0.949)$ between the two groups (Table 6). The median PFSs in the high-AEC (> $\left.0.045 \times 10^{9} / \mathrm{L}\right)$ and low-AEC $\left(\leq 0.045 \times 10^{9} / \mathrm{L}\right)$ groups were 168 and 116 days, respectively $(\mathrm{P}=0.0295$; Fig. 3).

\section{Discussion}

Immune checkpoint inhibitors such as anti-PD-1/PD-L1 inhibitors have become crucial therapeutic options for patients with advanced malignant tumors, but the associated irAEs may lead to treatment interruption or fatal disease [8-10]. Early prediction and correct treatment are critical for irAE management.

The relationships between irAEs and anti-PD-1/PD-L1 inhibitor responses in advanced malignant tumors have long been controversial. A recent meta-analysis of 30 studies showed that irAEs (especially endocrine, cutaneous, and low-grade irAEs) were significantly associated with PFS and overall survival in patients with advanced malignant tumors who were receiving anti-PD-1/PD-L1 inhibitors; however, that meta-analysis did not examine ORR [26]. In the present study, we found no significant differences in ORRs and DCRs between irAEs and no-irAEs groups, similar to previous results [22, 23], although an association between irAEs and PFS was not directly observed. Because of the immortal time bias of irAEs and the intersection points in the overall analysis, we used landmark analysis, in which the irAEs group showed a survival advantage after PFS for 120 days. This finding is related to the initial onset of irAEs: most irAEs 
reportedly appear within 3 months after the initiation of treatment, while serious adverse reactions such as immune-associated pneumonia appear within 2 months [27]. Our clinical data indicated that some patients discontinued treatment early because of severe adverse reactions such as immune-related myocardial injury and immune-related pneumonitis.

Peripheral blood markers such as baseline NLR and PLR have shown predictive value in the efficacy of anti-PD-1/PD-L1 inhibitors in advanced malignant tumors [28-33], as well as in the possibility of predicting the risk of irAE occurrence [12-14]. Moreover, eosinophils in peripheral blood were associated with irAEs [14-16]. In the present study, we assessed the predictive values of baseline NLR, PLR, and eosinophils for the risk of irAEs; we found that the incidences of irAEs were significantly higher in the baseline low-NLR and baseline high-AEC groups than in the high-NLR and low-AEC groups. Previous studies showed that higher baseline NLR predicted poor efficacy of anti-PD-1/PD-L1 inhibitors, indirectly suggesting the potential for a positive correlation between irAEs and treatment efficacy. Furthermore, univariate logistic analysis showed that both baseline low-NLR and baseline high-AEC were risk factors for irAEs; following correction for confounding factors (e.g., tumor type, treatment, and treatment line) multivariate logistic analysis showed that only AEC was an independent factor associated with irAEs. Baseline PLR reportedly can be used as an independent predictor of irAEs in the immune checkpoint inhibitor treatment of patients with advanced non-small cell lung cancer [12]; however, our multivariate analysis results did not support the above conclusion. We speculate that baseline AEC may have greater value in the prediction of irAE occurrence, compared with baseline NLR and PLR. To our knowledge, this is the first comparison of the predictive values of baseline NLR, baseline PLR, and baseline AEC for irAEs.

Accordingly, we investigated the relationship between baseline AEC and anti-PD-1/PD-L1 efficacy. We found no difference between high-AEC and low-AEC in terms of ORR, in contrast to the results of previous studies. However, we found that PFS was significantly better in the high-AEC group than in the low-AEC group [16].

Notably, we found that irAEs were more likely to occur in patients with good ECOG PS, similar to previous findings [20]. Following adjustment for confounding factors (e.g., tumor type, treatment, and treatment line), we found that good ECOG remained an independent positive predictor of irAEs. In addition, treatment lines are reportedly related to irAEs, such that second-line treatment and above is more likely to lead to irAEs [20], in contrast to our findings. In several studies, combination treatment of immunotherapy plus sequential or concurrent treatment with targeted therapy or cytotoxic chemotherapy has been reported to increase the risk of immune-related pneumonitis. Notably, we found that the incidence of irAEs was low in patients receiving immunotherapy combined with targeted therapy; because there are no other published data regarding irAEs in this patient population, additional large prospective studies of single tumor species are needed to confirm our findings.

There were a few limitations in this study. First, this was a single-center retrospective study. Second, this study may have underestimated the influences of hormones or immunosuppressants on irAE classification. Therefore, multi-center, prospective studies are needed to validate our results. 


\section{Conclusion}

Our findings indicate that baseline AEC and ECOG PS can be used as independent predictors of irAE occurrence to guide clinical practice, provide early warning of irAEs, and ensure preventive measures against irAE onset, thus aiding in the correct management of irAEs.

\section{Abbreviations}

ECOG, Eastern Cooperative Oncology Group

NLR, Neutrophil-lymphocyte ratio

PLR, Platelet-lymphocyte ratio

AEC, absolute eosinophil count

HR, Odds ratio

$\mathrm{Cl}$, confidence interval

irAEs, immune-related adverse events

ORR, Objective response rate

DCR, Disease control rate

ICls, Immune checkpoint inhibitor

$\mathrm{CR}$, Complete response

PR, Partial response,

SD, Stable disease

PD, Progressive disease

PFS, progression free survival

ROC, Receiver Operating Characteristic

ECOG, Eastern Cooperative Oncology Group,

PS, Performance status

\section{Declarations}




\section{Ethics approval and consent to participate}

The study protocol was approved by Beijing Friendship Hospital's Institutional Review Board (2020-P2176-01) and performed in accordance with the tenets of the Declaration of Helsinki.

\section{Consent for publication}

All presentations of case reports have consent to publish.

\section{Availability of data and materials}

The datasets generated and/or analysed during the current study are not publicly available due to personal privacy but are available from the corresponding author on reasonable request.

\section{Competing interests}

The authors declare that they have no competing interests.

\section{Funding}

This study was supported by the Capital Health Research and Development of Special, the Digestive Medical Coordinated Development Center of Beijing Hospitals Authority (No: XXT01), the Beijing Key Clinical Specialty, and the Pilot Project of Clinical Collaboration with Traditional Chinese Medicine and Western Medicine in Major Refractory Disease - Esophageal Cancer (2019-ZX-005).

\section{Authors' contributions}

All authors contributed to the study conception and design. All authors contributed to the study conception and design. Materials preparation and data collection were performed by Yan Ma, Xiao Ma, Jingting Wang, Jing Wang, and Bangwei Cao. Statistical support was provided by Shanshan Wu. The first draft of the manuscript was written by Yan $\mathrm{Ma}$, and the manuscript was critically revised and approved by all authors.

\section{Acknowledgements}

We thank all participants in the study.

\section{References}

1. Garon EB, Hellmann MD, Rizvi NA, Carcereny E, Leighl NB, Ahn M-J, et al. Five-Year Overall Survival for Patients With Advanced Non-Small-Cell Lung Cancer Treated With Pembrolizumab: Results From the Phase I KEYNOTE-001 Study. Journal Of Clinical Oncology. 2019;37(28):2518-2527.

2. Reck M, Rodriguez-Abreu D, Robinson AG, Hui R, Csoszi T, Fulop A, et al. Updated Analysis of KEYNOTE-024: Pembrolizumab Versus Platinum-Based Chemotherapy for Advanced Non-Small-Cell 
Lung Cancer With PD-L1 Tumor Proportion Score of 50\% or Greater. Journal Of Clinical Oncology. 2019;37(7):537-546.

3. Schmid P, Adams S, Rugo HS, Schneeweiss A, Barrios CH, Iwata H, et al. Atezolizumab and NabPaclitaxel in Advanced Triple-Negative Breast Cancer. New England Journal Of Medicine. 2018;379(22):2108-2121.

4. Sharma P, Retz M, Siefker-Radtke A, Baron A, Necchi A, Bedke J, et al. Nivolumab in metastatic urothelial carcinoma after platinum therapy (CheckMate 275): a multicentre, single-arm, phase 2 trial. Lancet Oncology. 2017;18(3):312-322.

5. Wolchok JD, Chiarion-Sileni V, Gonzalez R, Rutkowski P, Grob JJ, Cowey CL, et al. Overall Survival with Combined Nivolumab and Ipilimumab in Advanced Melanoma. New England Journal Of Medicine. 2017;377(14):1345-1356.

6. von Itzstein MS, Khan S, Gerber DE. Investigational Biomarkers for Checkpoint Inhibitor ImmuneRelated Adverse Event Prediction and Diagnosis. Clinical Chemistry. 2020;66(6):779-93.

7. Hellmann MD, Ciuleanu TE, Pluzanski A, Lee JS, Otterson GA, Audigier-Valette C, et al. Nivolumab plus Ipilimumab in Lung Cancer with a High Tumor Mutational Burden. New England Journal Of Medicine. 2018;378(22):2093-2104.

8. Champiat S, Lambotte O, Barreau E, Belkhir R, Berdelou A, Carbonnel F, et al. Management of immune checkpoint blockade dysimmune toxicities: a collaborative position paper. Annals Of Oncology. 2016;27(4):559-574.

9. Postow MA, Sidlow R, Hellmann MD. Immune-Related Adverse Events Associated with Immune Checkpoint Blockade. New England Journal Of Medicine. 2018;378(2):158-168.

10. Wang DY, Salem J-E, Cohen JV, Chandra S, Menzer C, Ye F, et al. Fatal Toxic Effects Associated With Immune Checkpoint Inhibitors A Systematic Review and Meta-analysis. Jama Oncology. 2018;4(12):1721-1728.

11. Couey MA, Bell RB, Patel AA, Romba MC, Crittenden MR, Curti BD, et al. Delayed immune-related events (DIRE) after discontinuation of immunotherapy: updates diagnostic hazard of autoimmunity at a distance. Journal for Immunotherapy Of Cancer. 2019;7.

12. Pavana A, Calvettid L, Dal Masoa A, Attilia I, Del Biancob P, Paselloa G, et al. Peripheral Blood Markers Identify Risk of Immune-Related Toxicity in Advanced Non-Small Cell Lung Cancer Treated with Immune-Checkpoint Inhibitors. Oncologist. 2019;24(8):1128-1136.

13. Peng L, Wang Y, Liu F, Qiu X, Zhang X, Fang C, et al. Peripheral blood markers predictive of outcome and immune-related adverse events in advanced non-small cell lung cancer treated with PD-1 inhibitors. Cancer Immunology Immunotherapy. 2020;69(9):1813-1822.

14. Fujisawa Y, Yoshino K, Otsuka A, Funakoshi T, Fujimura T, Yamamoto Y, et al. Fluctuations in routine blood count might signal severe immune-related adverse events in melanoma patients treated with nivolumab. Journal Of Dermatological Science. 2017;88(2):225-231.

15. Manson G, Norwood J, Marabelle A, Kohrt H, Houot R. Biomarkers associated with checkpoint inhibitors. Annals Of Oncology. 2016;27(7):1199-1206. 
16. Chu X, Zhao J, Zhou J, Zhou F, Jiang T, Jiang S, et al. Association of baseline peripheral-blood eosinophil count with immune checkpoint inhibitor-related pneumonitis and clinical outcomes in patients with non-small cell lung cancer receiving immune checkpoint inhibitors. Lung cancer (Amsterdam, Netherlands). 2020;150:76-82.

17. Freeman-Keller M, Kim Y, Cronin H, Richards A, Gibney G, Weber JS. Nivolumab in Resected and Unresectable Metastatic Melanoma: Characteristics of Immune-Related Adverse Events and Association with Outcomes. Clinical Cancer Research. 2016;22(4):886-894.

18. Haratani K, Hayashi H, Chiba Y, Kudo K, Yonesaka K, Kato R, et al. Association of Immune-Related Adverse Events With Nivolumab Efficacy in Non-Small Cell Lung Cancer. Jama Oncology. 2018;4(3):374-378.

19. Hua C, Boussemart L, Mateus C, Routier E, Boutros C, Cazenave H, et al. Association of Vitiligo With Tumor Response in Patients With Metastatic Melanoma Treated With Pembrolizumab. Jama Dermatology. 2016;152(1):45-51.

20. Riudavets M, Mosquera J, Garcia-Campelo R, Serra J, Anguera G, Gallardo P, et al. Immune-Related Adverse Events and Corticosteroid Use for Cancer-Related Symptoms Are Associated With Efficacy in Patients With Non-small Cell Lung Cancer Receiving Anti-PD-(L)1 Blockade Agents. Frontiers In Oncology. 2020;10.

21. Sato K, Akamatsu H, Murakami E, Sasaki S, Kanai K, Hayata A, et al. Correlation between immunerelated adverse events and efficacy in non-small cell lung cancer treated with nivolumab. Lung Cancer. 2018;115:71-74.

22. Teraoka $S$, Fujimoto $D$, Morimoto $T$, Kawachi $H$, Ito $M$, Sato $Y$, et al. Early Immune-Related Adverse Events and Association with Outcome in Advanced Non-Small Cell Lung Cancer Patients Treated with Nivolumab: A Prospective Cohort Study. Journal Of Thoracic Oncology. 2017;12(12):1798-1805.

23. Teulings H-E, Limpens J, Jansen SN, Zwinderman AH, Reitsma JB, Spuls PI, et al. Vitiligo-Like Depigmentation in Patients With Stage III-IV Melanoma Receiving Immunotherapy and Its Association With Survival: A Systematic Review and Meta-Analysis. Journal Of Clinical Oncology. 2015;33(7):773-781.

24. Arriola E, Wheater M, Galea I, Cross N, Maishman T, Hamid D, et al. Outcome and Biomarker Analysis from a Multicenter Phase 2 Study of Ipilimumab in Combination with Carboplatin and Etoposide as First-Line Therapy for Extensive-Stage SCLC. Journal Of Thoracic Oncology. 2016;11(9):1511-1521.

25. Rogado J, Sanchez-Torres JM, Romero-Laorden N, Ballesteros Al, Pacheco-Barcia V, Ramos-Levi A, et al. Immune-related adverse events predict the therapeutic efficacy of anti-PD-1 antibodies in cancer patients. European Journal Of Cancer. 2019;109:21-27.

26. Zhou X, Yao Z, Yang H, Liang N, Zhang X, Zhang F. Are immune-related adverse events associated with the efficacy of immune checkpoint inhibitors in patients with cancer? A systematic review and meta-analysis. Bmc Medicine. 2020;18(1).

27. Wu C-E, Yang C-K, Peng M-T, Huang P-W, Chang C-F, Yeh K-Y, et al. The association between immunerelated adverse events and survival outcomes in Asian patients with advanced melanoma receiving 
anti-PD-1 antibodies. Bmc Cancer. 2020;20(1).

28. Dharmapuri S, Ozbek U, Lin J-Y, Sung M, Schwartz M, Branch AD, et al. Predictive value of neutrophil to lymphocyte ratio and platelet to lymphocyte ratio in advanced hepatocellular carcinoma patients treated with anti-PD-1 therapy. Cancer Medicine. 2020;9(14):4962-4970.

29. Diem S, Schmid S, Krapf M, Flatz L, Born D, Jochum W, et al. Neutrophil-to-Lymphocyte ratio (NLR) and Platelet-to-Lymphocyte ratio (PLR) as prognostic markers in patients with non-small cell lung cancer (NSCLC) treated with nivolumab. Lung Cancer. 2017;111:176-181.

30. Jin J, Yang L, Liu D, Li W. Association of the neutrophil to lymphocyte ratio and clinical outcomes in patients with lung cancer receiving immunotherapy: a meta-analysis. Bmj Open. 2020;10(6).

31. Liu J, Li S, Zhang S, Liu Y, Ma L, Zhu J, et al. Systemic immune-inflammation index, neutrophil-tolymphocyte ratio, platelet-to-lymphocyte ratio can predict clinical outcomes in patients with metastatic non-small-cell lung cancer treated with nivolumab. Journal of Clinical Laboratory Analysis. 2019;33(8).

32. Matsuki T, Okamoto I, Fushimi C, Sawabe M, Kawakita D, Sato H, et al. Hematological predictive markers for recurrent or metastatic squamous cell carcinomas of the head and neck treated with nivolumab: A multicenter study of 88 patients. Cancer Medicine. 2020;9(14):5015-24.

33. Rossi S, Toschi L, Finocchiaro G, Santoro A. Neutrophil and lymphocyte blood count as potential predictive indicators of nivolumab efficacy in metastatic non-small-cell lung cancer. Immunotherapy. 2020;12(10):715-724.

\section{Tables}

Table 1. Patient characteristics $(n=95)$ 
Patient characteristics

\section{Age at initiation of anti-PD-1/PD-L1 therapy} (years)

Median

Range

\section{Sex}

Male

Female

ECOG

0

1

2

\section{Tumor type}

Lung cancer (NSCLC: $\mathrm{n}=20$, small cell lung cancer: $\mathrm{n}=5$ )

Esophageal carcinoma

Liver cancer

Head and neck cancer

Genital system cancer

Colorectal cancer

Gastric carcinoma

Urogenital carcinoma

Cutaneous soft tissue carcinoma

Melanoma

Gallbladder carcinoma and bile duct carcinoma

Others

\section{TNM clinical classification}

III

29 (30.5)

IV

58 (61.1)

Unknown
Patients treated with anti-PD-1/PD-L1 therapy ( $\mathrm{n}=$ 95), n (\%)

62

$30-80$

66 (69.5)

$29(30.5)$

$25(26.3)$

$61(64.2)$

$9(9.5)$

$25(26.3)$

17 (17.9)

11 (11.6)

8 (8.4)

$6(6.3)$

7 (7.4)

7 (7.4)

4 (4.2)

3 (3.2)

2 (2.1)

2 (2.1)

3 (3.2) 


\begin{tabular}{|ll|}
\begin{tabular}{|l|} 
Treatment lines at initiation of anti-PD-1/PD-L1 \\
therapy
\end{tabular} & $36(37.9)$ \\
First-line & $29(30.5)$ \\
Second-line & $30(31.6)$ \\
Third-line and above & \\
\hline Treatment & $38(40)$ \\
Immunotherapy & $22(23.2)$ \\
Immunotherapy + targeted therapy & $31(32.6)$ \\
Immunotherapy + chemotherapy & $4(4.2)$ \\
Immunotherapy + chemotherapy + targeted & \\
therapy & \\
\hline Baseline ACE & $0.12 \pm 0.017$ \\
Mean \pm SD & \\
\hline Baseline PLR & $204.899 \pm 102.712$ \\
\hline Mean \pm SD & \\
\hline Baseline NLR & 3.381 \\
Median & $1.021-40.625$ \\
Range & \\
\hline
\end{tabular}

Table 2 Description of irAEs occurring in individual patients 


\begin{tabular}{|c|c|}
\hline irAEs category & Number of patients with irAEs (\%) \\
\hline \multicolumn{2}{|l|}{ Cutaneous } \\
\hline$\underline{\text { Rash }}$ & $8(8.4 \%)$ \\
\hline Pruritus & $2(2.1 \%)$ \\
\hline Vitiligo & $1(1.0 \%)$ \\
\hline Reactive cutaneous capillary endothelial proliferation & $5(5.2 \%)$ \\
\hline \multicolumn{2}{|l|}{ Endocrine-related events } \\
\hline Hypothyroidism & $3(3.1 \%)$ \\
\hline Diabetes & $1(1.0 \%)$ \\
\hline \multicolumn{2}{|l|}{ Hepatotoxicity } \\
\hline$\underline{\text { ALT/AST elevation }}$ & $11(11.6 \%)$ \\
\hline \multicolumn{2}{|l|}{ Gastrointestinal toxicity } \\
\hline Diarrhea & $3(3.1 \%)$ \\
\hline Gastrointestinal bleeding & $1(1.0 \%)$ \\
\hline Immune-associated pneumonia & $7(7.3 \%)$ \\
\hline Cardiac toxicity & $5(5.2 \%)$ \\
\hline \multicolumn{2}{|l|}{ Hematological toxicity } \\
\hline Leukopenia & $4(4.2 \%)$ \\
\hline Thrombocytopenia & $4(4.2 \%)$ \\
\hline Anemia & $7(7.3 \%)$ \\
\hline \multicolumn{2}{|l|}{ Others } \\
\hline Increased creatinine & $2(2.1 \%)$ \\
\hline Peripheral neuropathy. & $2(2.1 \%)$ \\
\hline Shingles & $1(1.0 \%)$ \\
\hline Thromboembolism & $1(1.0 \%)$ \\
\hline Hippocampal inflammation & $1(1.0 \%)$ \\
\hline Fatigue & $3(3.1 \%)$ \\
\hline Amylase and lipase elevation & $1(1.0 \%)$ \\
\hline Oral mucositis & $1(1.0 \%)$ \\
\hline
\end{tabular}


(Note: The mechanism of cardiotoxicity associated with ICls is not yet fully understood. Cardiotoxicity here mainly includes myocarditis, heart failure, or myocardial infarction. Abbreviation: irAEs, immunerelated adverse events.)

Table 3. ORRs and DCRs of irAEs and no-irAEs groups

\begin{tabular}{|cccccc|}
\hline & \multicolumn{4}{c}{ irAEs $(n=53)$} & \multicolumn{2}{l}{ no-irAEs $(n=42)$} & P-value \\
& $n$ & $\%$ & $n$ & $\%$ & \\
ORR & 7 & 13.2 & 5 & 11.9 & $0.763^{\text {a }}$ \\
\cline { 1 - 2 } DCR & 32 & 60.4 & 29 & 69.0 & $0.381^{\text {b }}$ \\
\hline
\end{tabular}

( ${ }^{a}$ continuity correction; b Pearson chi-squared. Abbreviations: irAEs, immune-related adverse events; ORR, objective response rate; $D C R$, disease control rate)

Table 4. Associations between peripheral blood markers and irAEs

\begin{tabular}{|c|c|c|c|}
\hline Blood parameter & Cutoff value & irAEs, n (\%) & P-value \\
\hline NLR & 8.58 & & $0.041^{*}$ \\
\hline Low $(n=86)$ & & $51 / 86(59.3 \%)$ & \\
\hline $\operatorname{High}(\mathrm{n}=9)$ & & $2 / 9(22.2 \%)$ & \\
\hline PLR & 180.68 & & 0.089 \\
\hline Low $(n=50)$ & & $32 / 50(64 \%)$ & \\
\hline High $(n=45)$ & & $21 / 45$ (46.7\%) & \\
\hline AEC & $0.045 \times 10^{9} / \mathrm{L}$ & & $0.010 *$ \\
\hline Low $(n=22)$ & & $7 / 22(31.8 \%)$ & \\
\hline High $(n=73)$ & & $46 / 73(63 \%)$ & \\
\hline
\end{tabular}

(*P < 0.05. Abbreviations: NLR, neutrophil-lymphocyte ratio; PLR, platelet-lymphocyte ratio; AEC, absolute eosinophil count) 
Table 5. Univariate and multivariate logistic regression analyses of irAEs 


\begin{tabular}{|c|c|c|c|c|c|c|}
\hline & \multicolumn{3}{|c|}{ Univariate analyses } & \multicolumn{3}{|c|}{ Multivariate analyses } \\
\hline & P-value & OR & $95 \% \mathrm{Cl}$ & $\begin{array}{l}\mathrm{P}- \\
\text { value }\end{array}$ & OR & $95 \% \mathrm{Cl}$ \\
\hline Sex & 0.330 & 0.646 & $\begin{array}{l}0.268- \\
1.555\end{array}$ & - & - & - \\
\hline Age & 0.975 & 1.001 & $\begin{array}{l}0.957- \\
1.046\end{array}$ & - & - & - \\
\hline ECOG & $0.0499 *$ & 0.507 & $\begin{array}{l}0.241- \\
1.065\end{array}$ & $0.046^{*}$ & 0.159 & $\begin{array}{l}0.026- \\
0.970\end{array}$ \\
\hline \multicolumn{7}{|l|}{$0-1^{c}$} \\
\hline \multicolumn{7}{|l|}{2} \\
\hline Tumor type & 0.795 & 1.018 & $\begin{array}{l}0.892- \\
1.161\end{array}$ & 0.770 & - & - \\
\hline TNM clinical classification & 0.508 & 0.786 & $\begin{array}{l}0.385- \\
1.605\end{array}$ & - & - & - \\
\hline Treatment line & 0.150 & 1.939 & $\begin{array}{l}0.787- \\
4.777\end{array}$ & 0.69 & 2.908 & $\begin{array}{l}0.922- \\
9.169\end{array}$ \\
\hline \multicolumn{7}{|l|}{ First-line and second-line ${ }^{c}$} \\
\hline \multicolumn{7}{|l|}{ Third-line and above } \\
\hline Treatment & 0.125 & 0.780 & $\begin{array}{l}0.567- \\
1.072\end{array}$ & $0.044^{\star}$ & - & - \\
\hline Immunotherapy ${ }^{c}$ & & & & Base & - & - \\
\hline Immunotherapy + targeted therapy & & & & $0.005^{\star}$ & 0.156 & $\begin{array}{l}0.045- \\
0.544\end{array}$ \\
\hline Immunotherapy + chemotherapy & & & & 0.301 & 0.550 & $\begin{array}{l}0.178- \\
1.706\end{array}$ \\
\hline $\begin{array}{l}\text { Immunotherapy + chemotherapy + } \\
\text { targeted therapy }\end{array}$ & & & & 0.383 & 0.363 & $\begin{array}{l}0.037- \\
3.533\end{array}$ \\
\hline NLR & $0.0499 *$ & 0.196 & $\begin{array}{l}0.038- \\
1.000\end{array}$ & 0.505 & 0.501 & $\begin{array}{l}0.066- \\
3.816\end{array}$ \\
\hline \multicolumn{7}{|l|}{$\operatorname{Low}(\leq 8.58)^{c}$} \\
\hline \multicolumn{7}{|l|}{ High (> 8.58) } \\
\hline PLR & 0.091 & 0.492 & $\begin{array}{l}0.216- \\
1.120\end{array}$ & 0.216 & 0.537 & $\begin{array}{l}0.200- \\
1.440\end{array}$ \\
\hline \multicolumn{7}{|l|}{$\operatorname{Low}(<180.68)^{c}$} \\
\hline \multicolumn{7}{|l|}{$\operatorname{High}(\geq 180.68)$} \\
\hline AEC & $0.012^{\star}$ & 3.651 & $\begin{array}{l}1.322- \\
10.076\end{array}$ & $0.014^{\star}$ & 4.114 & $\begin{array}{l}1.32- \\
12.858\end{array}$ \\
\hline
\end{tabular}


$\operatorname{Low}\left(\leq 0.045 \times 10^{9} / \mathrm{L}\right)^{\mathrm{c}}$

$\operatorname{High}\left(>0.045 \times 10^{9} / \mathrm{L}\right)$

( ${ }^{\star} \mathrm{P}<0.05 .{ }^{\mathrm{C}}$ comparison reference in multivariate analyses. Abbreviations: ECOG, Eastern Cooperative Oncology Group; NLR, neutrophil-lymphocyte ratio; PLR, platelet-lymphocyte ratio; AEC, absolute eosinophil count; OR, odds ratio; $\mathrm{Cl}$, confidence interval)

Table 6. ORRs and DCRs of high-AEC and low-AEC groups

\begin{tabular}{|cccccc|}
\hline \multicolumn{5}{|c}{ High-AEC $(\mathrm{n}=73)$} & \multicolumn{2}{c|}{ Low-AEC $(\mathrm{n}=22)$} & P-value \\
& $\mathrm{n}$ & $\%$ & $\mathrm{n}$ & $\%$ & \\
ORR & 7 & 9.5 & 5 & 22.7 & $0.208^{\mathrm{a}}$ \\
\cline { 1 - 2 } & 47 & 64.3 & 14 & 63.6 & $0.949^{\mathrm{b}}$ \\
\hline
\end{tabular}

( ${ }^{a}$ continuity correction, ${ }^{b}$ Pearson chi-squared. Abbreviations: AEC, absolute monocyte count; ORR, objective response rate; DCR, disease control rate)

\section{Figures}

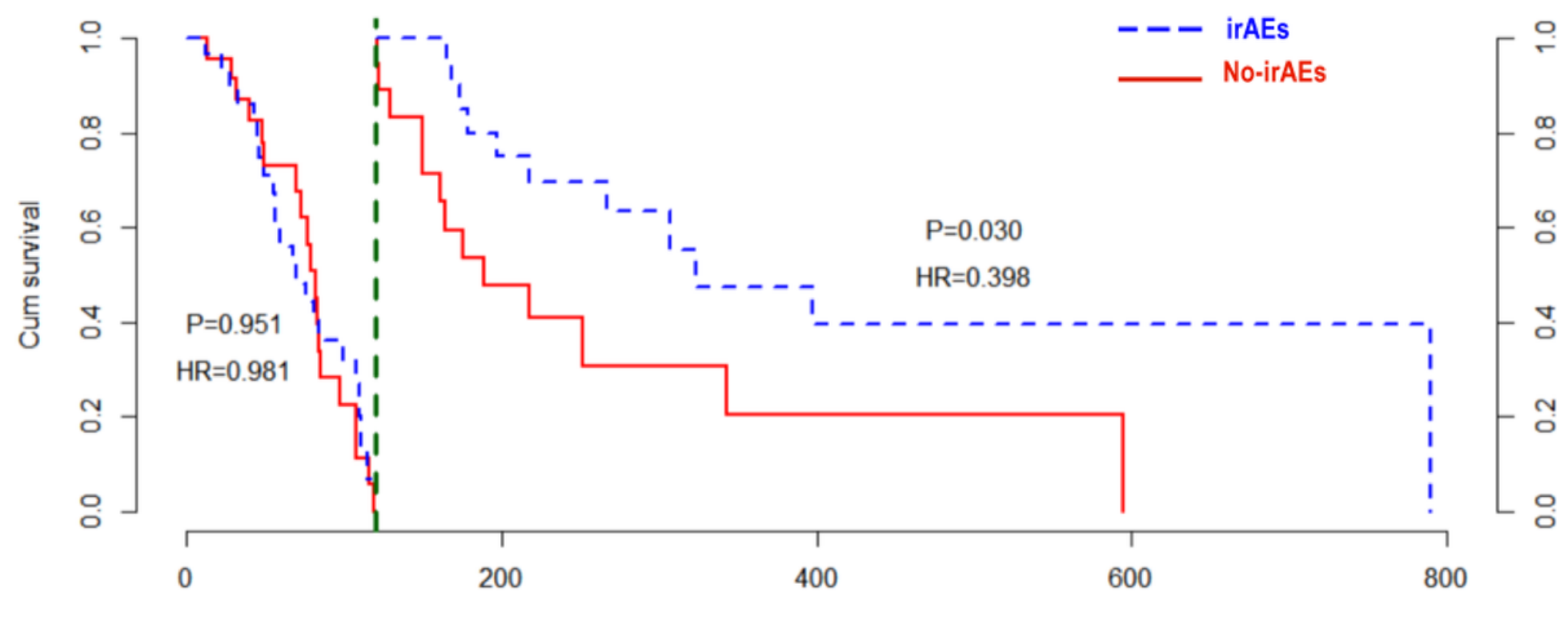

Progression free survival(days)

Figure 1 
Landmark analysis according to the presence of irAEs. Kaplan-Meier curves with the threshold of 120 days (landmark analysis) for progression-free survival. Abbreviations: irAEs, immune-related adverse events; HR, hazard ratio.

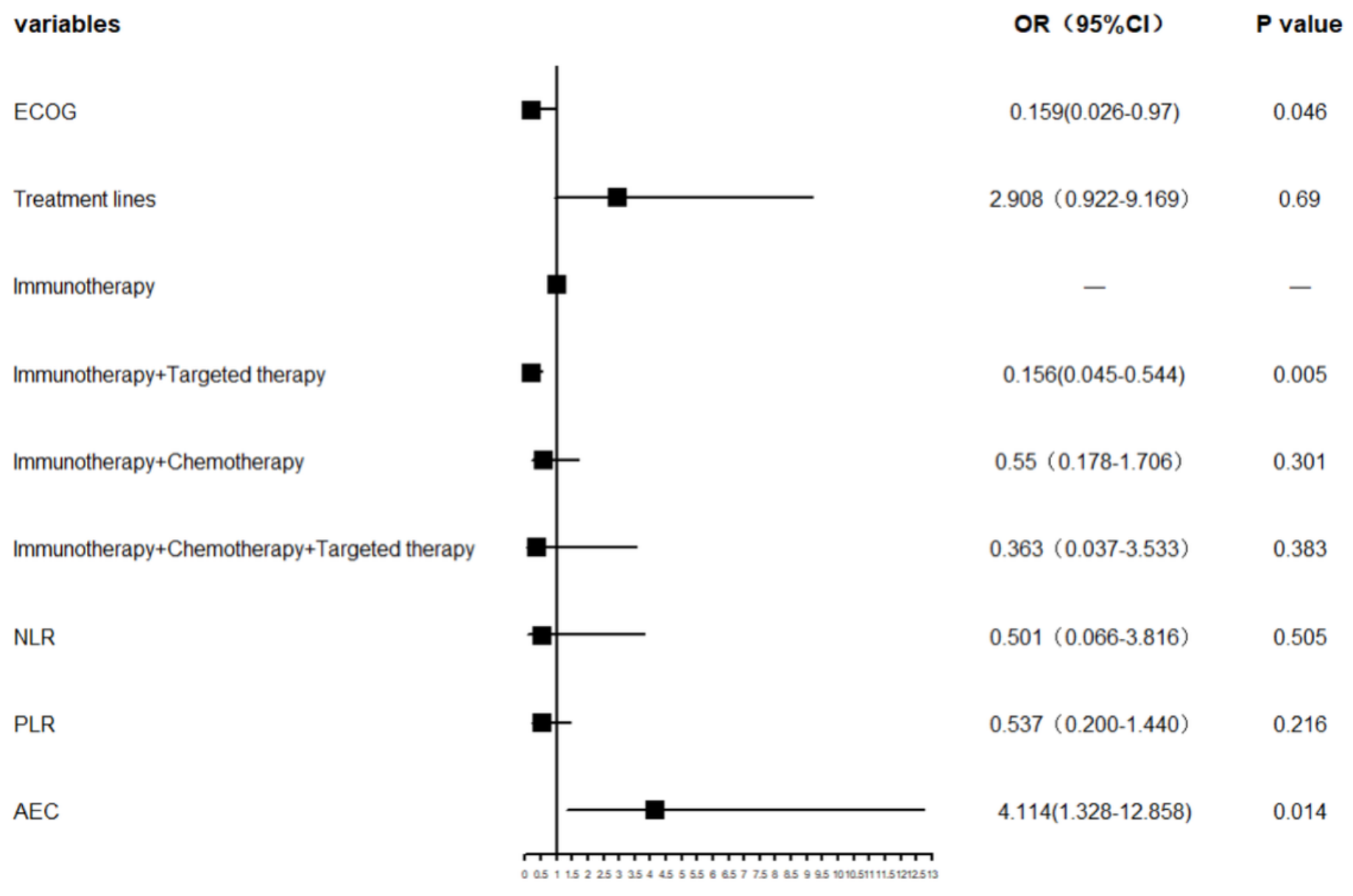

\section{Figure 2}

Forest plot of multivariate logistic regression analyses for irAEs. Vertical line in the middle of the figure is the invalid line (i.e., OR: = 1). Each horizontal line is the line between the upper and lower limits of $95 \% \mathrm{Cl}$ of the study; the length of each line segment intuitively represents the size of $95 \% \mathrm{Cl}$. Small square in the center of the horizontal segment is the position of the OR value; its size reflects the weight of the study. The reference of comparative subgroups was the same as the multivariate logistic analysis of predictive markers for irAEs. Abbreviations: ECOG, Eastern Cooperative Oncology Group; NLR, neutrophil-lymphocyte ratio; PLR, platelet-lymphocyte ratio; $\mathrm{AEC}$, absolute eosinophil count; $\mathrm{OR}$, odds ratio; $\mathrm{Cl}$, confidence interval. 


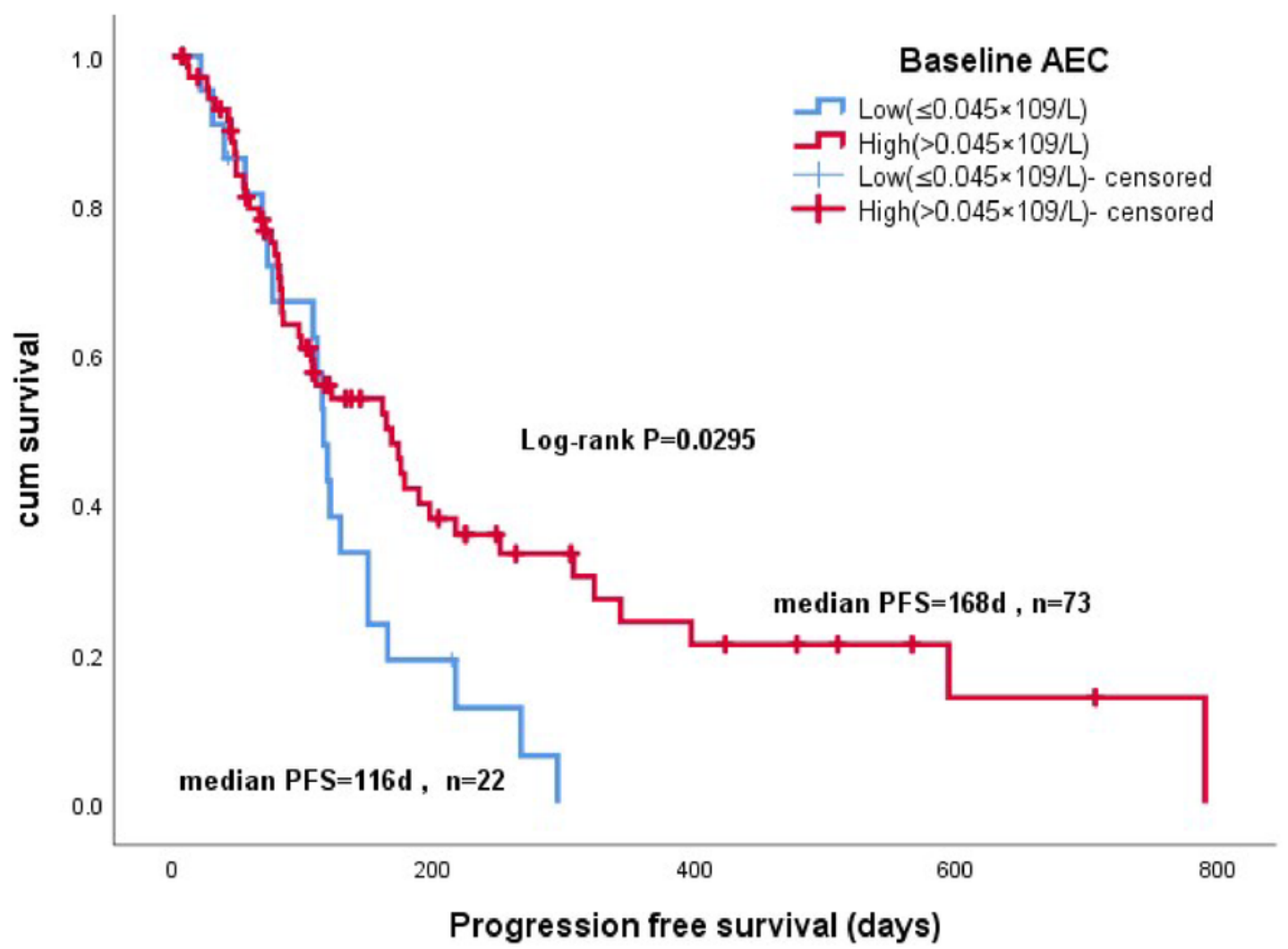

Figure 3

Kaplan-Meier survival curves for progression-free survival stratified according to baseline AEC cutoff, determined by ROC curve analysis. 